\title{
Steering distillation processes through quantum Zeno dynamics
}

\author{
B. Militello, ${ }^{1}$ H. Nakazato, ${ }^{2}$ and A. Messina ${ }^{1}$ \\ ${ }^{1} I N F M$, MIUR, and Dipartimento di Scienze Fisiche ed Astronomiche dell'Università di Palermo, \\ Via Archirafi, 36, I-90123 Palermo, Italy \\ ${ }^{2}$ Department of Physics, Waseda University, Tokyo 169-8555, Japan
}

(Received 18 April 2004; published 8 March 2005)

\begin{abstract}
A quantum system in interaction with a repeatedly measured one undergoes a nonunitary time evolution and is pushed into a subspace substantially determined by the two-system coupling. The possibility of suitably modifying such an evolution through quantum Zeno dynamics (i.e., the generalized quantum Zeno effect) addressing the system toward an a priori decided target subspace is illustrated. Applications and their possible realizations in the context of trapped ions are also discussed.
\end{abstract}

DOI: 10.1103/PhysRevA.71.032102

PACS number(s): 03.65.Xp, 03.65.Ta, 32.80.Pj

\section{INTRODUCTION}

A distillation process, in its essence, is nothing but a systematic way of driving a system toward a state nonorthogonal to its initial condition. In some sense, thus, it often represents the realization of a projection operator. In recent years, efforts have been made to realize distillation procedures since they might be exploited to prepare and to control at one's will the state of a quantum system. In the field of quantum technology $[1,2]$ covering such research areas as quantum computation, quantum information and quantum teleportation, it is usually assumed that a desired state, which is necessary to start a specific quantum manipulation, can be prepared in principle. In reality, however, it is not at all trivial and we have to specify explicitly how such a preparation is actually implemented. Various distillation processes have been proposed for this purpose so far $[1,2]$.

Recently a general strategy of distillation specifically addressed to bipartite systems has been reported [3]. The key idea of this distillation process is to repeatedly measure a system (here called master and denoted by $M$ ) interacting with another one (here called slave and denoted by $S$ ) provoking a nonunitary evolution which forces the latter toward a subspace determined by the specific $M-S$ coupling and by the results of the master measurements. It occurs with a probability that expresses the efficiency of the process.

Such a scheme for distillation shares some points with quantum nondemolition measurement (QND) methods [4,5], also based on the idea of repeatedly measuring a system in interaction with the one we want to observe. It is worth mentioning that, although the original scope of the QND was to measure observables (let us denote by $A_{S}$ the generic one) related to the nonrepeatedly measured system (corresponding to the slave in the distillation scheme), the procedure (being nothing but a practical realization of a measurement on the slave) has the collateral effect of projecting the slave onto an eigenspace of the measured observable. Therefore, QND provides a way to generate quantum states through their $e x$ traction from the initial condition of the relevant physical system (i.e., the slave) [6-8]. Despite these similarities between distillation and QND, the formalism introduced in Ref. [3] allows one to bring to light all the potentialities of such a scheme overcoming restrictions present in the stan- dard description of QND. Indeed, while in the quantum nondemolition measurement approach it is required that the ana$\log$ of the $M-S$ coupling should commute with the slave observable one is going to measure (a sufficient condition to realize a projection onto $A_{S}$ subspaces), in distillation schemes a necessary and sufficient condition on the nonunitary operator describing the nonunitary dynamics above mentioned may be directly given in order to establish which subspace is going to be extracted, that is, distilled.

The interest in such a process is twofold. On the one hand, as already mentioned, it allows the realization of applications in the context of quantum state manipulation and in particular quantum state preparation. On the other hand, it is also interesting in fundamental physics, describing the behavior of a physical system when a part of it is repeatedly measured.

In this paper we analyze in depth the distillation process reported in [3], bringing to light the close connection between the final result of the distillation process and the master-slave interaction. In particular, neglecting any environment effect, we show that the survival probabilities of the slave states under the unitary evolution due to the $M-S$ interaction are the crucial elements of this process. We shall prove that the higher is such a unitary survival probability, the higher is the probability that the relevant slave state is distilled by the process. On the basis of this observation, we demonstrate the possibility of steering distillation processes using mechanisms of control of survival probability relying on the generalized quantum Zeno effect, i.e., the quantum Zeno dynamics [9-12] recalled in the following.

In the next section, we recall the general statement of repeated measurement-based distillation following Ref. [3], and show applications in the specific case wherein the master is a two-level system. In the third section, we analyze the nature of the distillation and point out its connection with the survival probabilities related to the unitary evolution induced by the $M-S$ interaction. In the same section, we recall the quantum Zeno dynamics and show how to use it for controlling such survival probabilities and hence for steering distillation. The realizations of some classes of projection operators in a harmonic-oscillator Hilbert space are reported in the case where the master is a few-level system. The realization of operators projecting in finite or infinite dimensional sub- 
spaces corresponding to finite or infinite ranges of energy is described. In the final section, we discuss and show the possibility of realizing the method presented, in the context of trapped ions, and give some concluding remarks.

\section{DISTILLATION PROCESSES}

In this section, we analyze the behavior of a compound system when a part of it is repeatedly measured. It is easily understood that the measurements generally introduce nonunitary elements in the time evolution, and provoke the decay of a subset (subspace) of slave states when certain conditions are satisfied. In this circumstance, a projection operator onto a slave subspace is practically realized. The required conditions concern the spectrum of the nonunitary operator responsible for the evolution. As will be reported at the end of this section, in the case wherein the master is a two-level system, the relevant nonunitary operator is shown to be diagonalized and such conditions are explicitly examined. Moreover, it is necessary to analyze the relevant spectrum to determine the duration of the unitary interaction (i.e., the interval between adjacent measurements) and to individuate the classes of distillable subspaces.

\section{A. General statement}

Consider two interacting systems, referred to as master $(M)$ and slave $(S)$. Let $\left\{\left|\phi_{k}\right\rangle\right\}$ denote a basis of $M$, and $\left\{\left|\varphi_{n}\right\rangle\right\}$ indicate a basis of $S$. Let us denote by $\hat{U}(\tau)=e^{-i \hat{H} \tau}$ the time evolution operator $(\hbar=1)$ for time $\tau, \hat{H}$ being the compound system Hamiltonian.

Perform a measurement of the master state, and assume that the result is $\left|\phi_{0}\right\rangle$. Let the system evolve for time $\tau$ under $\hat{H}$, then perform another measurement of the master, and so on $N$ times. Assume that at each step the master system is found in the state $\left|\phi_{0}\right\rangle$. In such a case, the compound system is subjected to a nonunitary time evolution characterized by the operator

$$
\hat{W}^{(N)}(\tau) \equiv \mathcal{N}_{N}\left[\left|\phi_{0}\right\rangle\left\langle\phi_{0}\right| \hat{U}(\tau)\right]^{N}\left|\phi_{0}\right\rangle\left\langle\phi_{0}\left|=\mathcal{N}_{N}\right| \phi_{0}\right\rangle\left\langle\phi_{0}\right|[\hat{V}(\tau)]^{N},
$$

where

$$
\hat{V}(\tau) \equiv\left\langle\phi_{0}\left|e^{-i \hat{H} \tau}\right| \phi_{0}\right\rangle
$$

is a nonunitary operator describing the transformation that the slave state undergoes owing to both the time evolution $\hat{U}(\tau)$ and the projection following the successful measurement act on $M$. The normalization factor $\mathcal{N}_{N}$ takes into account the probability of finding $M$ in the state $\left|\phi_{0}\right\rangle N$ times.

Let us solve the eigenvalue problem related to $\hat{V}(\tau)$. Since this operator is not Hermitian, generally its right and left eigenvalue problems have different solutions. Assume now that it is possible to diagonalize the operator $\hat{V}(\tau)$ in the standard way [13]

$$
\hat{V}(\tau)=\sum_{k} \gamma_{k} \hat{P}_{k}
$$

where $\left\{\hat{P}_{k}\right\}$ are orthogonal projection operators satisfying the completeness relation $\Sigma_{k} \hat{P}_{k}=\hat{1}_{S}, \hat{1}_{S}$ being the slave identity operator, and $\gamma_{k}$ are complex numbers having moduli in general not greater than 1 . We shall denote also by $\left|\gamma_{k}, l\right\rangle$ the eigenstates corresponding to $\hat{P}_{k}, l$ being indices spanning the eventually degenerate eigenspace, so that $\hat{P}_{k}=\Sigma_{l}\left|\gamma_{k}, l\right\rangle\left\langle\gamma_{k}, l\right|$.

Introducing $\gamma=\max _{k}\left\{\left|\gamma_{k}\right|\right\}$, one finds, for large enough $N$,

$$
\hat{W}^{(N)}(\tau) \approx \aleph_{N}\left|\phi_{0}\right\rangle\left\langle\phi_{0}\right| \sum_{k:\left|\gamma_{k}\right|=\gamma} \gamma_{k}^{N} \hat{P}_{k}
$$

which may also be cast in the form

$$
\hat{W}^{(N)}(\tau) \approx \aleph_{N}\left|\phi_{0}\right\rangle\left\langle\phi_{0}\right| \gamma^{N} \hat{R}^{N} \hat{P}_{\text {dist }}
$$

where $\hat{R}:=\sum_{k:\left|\gamma_{k}\right|=\gamma}\left(\gamma_{k} / \gamma\right) \hat{P}_{k}$ (and hence $\hat{R}^{N}$ ) is a unitary operator acting on the distilled slave subspace corresponding to the projection operator

$$
\hat{P}_{d i s t}:=\sum_{k:\left|\gamma_{k}\right|=\gamma} \hat{P}_{k} .
$$

If a unique eigenvalue of $\hat{V}(\tau)$ having modulus $\gamma$ exists and in addition its eigenspace is nondegenerate, then the distillation procedure realizes just a single-state selection.

It deserves to be noted that the distillation is a conditional procedure in the sense that its success depends on $N$ measurement results. More precisely, the master system $(M)$, at each measurement act, should be always found in the state $\left|\phi_{0}\right\rangle$. Moreover, the procedure substantially realizes a projection operator; hence, if the process is successful, the would-be distilled states should be present in the initial slave state $\hat{\rho}_{s}$, where the initial state of the compound system is assumed to be $\hat{\rho}_{s} \otimes\left|\phi_{0}\right\rangle\left\langle\phi_{0}\right|$. The joint probability of finding the master system in its initial state $\left|\phi_{0}\right\rangle$ at each step $\left(\Pi_{k=1}^{N} \wp_{k}^{(M)}, \wp_{k}^{(M)}\right.$ being the probability of finding the master in $\left|\phi_{0}\right\rangle$ at the $k$ th measurement) tends, in the limit $N \rightarrow \infty$ and in the case $\gamma=1$, to the probability of finding the target subspace (i.e., the "distillate") into the initial slave state [17] (see the Appendix), that is,

$$
\prod_{k=1}^{N} \wp_{k}^{(M)} \rightarrow \operatorname{Tr}_{S}\left\{\hat{\rho}_{s} \hat{P}_{d i s t}\right\}
$$

where $\operatorname{Tr}_{S}$ is the trace operation over the slave degrees of freedom.

The quantity in Eq. (7) expresses the efficiency of the distillation process, that is, the success probability of distillation. In connection with this point, it is worth remarking that in the case $\gamma=1$ the distillation may be thought of as a simulation of a measurement act on the slave. In fact, it projects the system onto a prefixed subspace with a probability given by the norm of the component of the slave state onto the distilled subspace.

Incidentally, Eq. (7) explains also the fact that QND may be used in many physical systems, for instance in trapped ions, both as a strategy for generating states and for measur- 
ing slave state populations [6-8]. Indeed, on the one hand such processes project the system onto the distilled subspace with an efficiency given by Eq. (7) (state generation). On the other hand, the master is found in the state $\left|\phi_{0}\right\rangle$ all $N$ times with a joint probability which (for large enough $N$ ) is equal to the population of the target subspace in the initial slave state, providing a way to measure such populations (population measurements).

\section{B. A case of spin 1/2 as the master}

In general, the eigenvalue problem of $\hat{V}(\tau)$ is not trivial, in the sense that the left and right problems are not adjoint to each other. As an example of solvable models, let us consider the case wherein the master is a two-level system (formally of spin $\frac{1}{2}$ ), while the slave is a harmonic oscillator. This is not just an academic example. Indeed, in the context of trapped ions, it is possible to realize a wide variety of such couplings provided the experimental setup is adequately adjusted. In fact, the actions of suitably tuned and polarized laser fields on the confined atom are responsible for specific vibronic couplings (vibronic, i.e., involving both the internal and center-of-mass motional degrees of freedom) of the form [14-16]

$$
\hat{H}_{v}=\varepsilon \hat{\Omega} \hat{\sigma}_{+}+\text {H.c. }
$$

where $\hat{\sigma}_{+}=|+\rangle\langle-|\left(\hat{\sigma}_{-}=|-\rangle\langle+|\right)$is the Pauli raising (lowering) operator, $| \pm\rangle$ being the internal ionic states, and $\varepsilon$ is a positive coupling constant related to laser intensities and initial phases. The generic time-independent operator $\hat{\Omega}$ acts in the slave Hilbert space, and its specific form is determined once the specific nature of the master-slave interaction is given [17].

Assume that the master $(M)$ is initially in the state $|+\rangle$, hence starting with the density operator

$$
\hat{\rho}=\hat{\rho}_{v} \otimes|+\rangle\langle+|,
$$

$\hat{\rho}_{v}$ being the initial slave state. Let the system evolve under the action of the Hamiltonian $\hat{H}_{v}(M-S$ interaction) for time $\tau$, and then measure the internal ionic state. Assume the system is found in $|+\rangle$, and then let again the system evolve in accordance with $\hat{H}_{v}$ for another time $\tau$, and measure the fermionic state finding it in $|+\rangle$, and so on $N$ times.

Under these assumptions, the system undergoes the nonunitary evolution essentially described by the $N$ th power of the nonunitary operator

$$
\hat{V}(\tau) \equiv\left\langle+\left|e^{-i \hat{H}_{v} \tau}\right|+\right\rangle=\cos \left(\varepsilon \tau \sqrt{\hat{\Omega} \hat{\Omega}^{\dagger}}\right) .
$$

Such an explicit expression provides a very useful insight for forecasting the result of a distillation based upon interactions of the form (8). Indeed, denoting by $\omega_{k}$ and $\left|\omega_{k}\right\rangle$ the eigenvalues and eigenstates of the operator $\hat{\Omega} \hat{\Omega}^{\dagger}$, it is possible to distill a class of eigenstates simply by adjusting the pulse area $\varepsilon \tau$. In fact, for large enough $N$, it turns out that

$$
\cos ^{N}\left(\varepsilon \tau \sqrt{\omega_{k}}\right)=(-1)^{l_{k} N} \quad \text { if } \varepsilon \tau \sqrt{\omega_{k}}=l_{k} \pi,
$$

$$
\cos ^{N}\left(\varepsilon \tau \sqrt{\omega_{k}}\right) \approx 0 \text { otherwise, }
$$

and hence, choosing, for a prefixed $\bar{\omega}$, the pulse area $\varepsilon \tau$ such that for instance $\varepsilon \tau \sqrt{\bar{\omega}}=\pi$, it is possible to distill all those states belonging to integer-squared multiples of $\bar{\omega}$, and if no such eigenvalues exist, only the state corresponding to $\bar{\omega}$ is distilled.

In the context of trapped ions, this result may be used to realize a wide variety of applications concerning the generation of states whose Fock statistics involves only Fock states corresponding to perfect-square numbers, the generation of common eigenstates of energy and angular momentum, angular momentum Schrödinger cat states, and so on [17].

\section{CONTROLLING DISTILLATION THROUGH QUANTUM ZENO EFFECT}

In this section, we explain the connection between the final result of the distillation and the $M-S$ interaction, showing that the distilled subspace is generated by those slave states which undergo unitary evolutions, in the time $\tau$, characterized by the highest survival probability. On the basis of this fact, we propose to use quantum Zeno effect to control such survival probabilities. Moreover we show some applications, in particular the realization of what we call high-, low-, and bandpass bosonic filters, i.e., projection operators on subspaces whose states possess excitation numbers higher or lower than a quantity, or in a range.

\section{A. Unitary vs nonunitary survival probability}

Consider the orthonormal basis of the $M-S$ system $\left|\gamma_{k}, l\right\rangle\left|\phi_{j}\right\rangle \equiv\left|\gamma_{k}, l\right\rangle \otimes\left|\phi_{j}\right\rangle$, and denote by $\left|\psi_{k l, j}(\tau)\right\rangle$ its evolution induced by $\hat{U}(\tau):\left|\psi_{k l, j}(\tau)\right\rangle \equiv \hat{U}(\tau)\left|\gamma_{k}, l\right\rangle\left|\phi_{j}\right\rangle$. Therefore, the evolution operator may be cast in the form

$$
\hat{U}(\tau)=\sum_{k, l, j}\left|\psi_{k l, j}(\tau)\right\rangle\left\langle\gamma_{k}, l\right|\left\langle\phi_{j}\right| .
$$

Since $\hat{V}(\tau) \equiv\left\langle\phi_{0}|\hat{U}(\tau)| \phi_{0}\right\rangle=\Sigma_{k, l} \gamma_{k}\left|\gamma_{k}, l\right\rangle\left\langle\gamma_{k}, l\right|$, we obtain $\left\langle\phi_{0} \mid \psi_{k l, 0}(\tau)\right\rangle=\gamma_{k}\left|\gamma_{k} l\right\rangle$. This means that $\hat{U}(\tau)\left|\gamma_{n}, l\right\rangle\left|\phi_{0}\right\rangle$ $=\sqrt{\wp_{n}(\tau)} e^{i \xi_{n}(\tau)}\left|\gamma_{n}, l\right\rangle \otimes\left|\phi_{0}\right\rangle+\Sigma_{j \neq 0} \Sigma_{m, q} c_{j, m q}(\tau)\left|\gamma_{m}, q\right\rangle \otimes\left|\phi_{j}\right\rangle$, $\wp_{n}(\tau)$ being the relevant survival probability related to $\gamma_{n}$ through $\gamma_{n}=\sqrt{\wp_{n}(\tau)} e^{i \xi_{n}(\tau)}$. Therefore one immediately finds

$$
\hat{V}(\tau)=\sum_{n, l} \sqrt{\wp_{n}(\tau)} e^{i \xi_{n}(\tau)}\left|\gamma_{n}, l\right\rangle\left\langle\gamma_{n}, l\right| .
$$

Such a decomposition gives a precise physical meaning to the moduli of the eigenvalues of $\hat{V}(\tau)$ and shows that the distilled (preserved) slave states (corresponding to higher eigenvalue moduli) are those undergoing unitary evolutions (between two measurement acts) which do not induce (or induce smaller) abandon of the initial master states $\left|\phi_{0}\right\rangle$ in favor of the others $\left|\phi_{j}\right\rangle, j \neq 0$. In other words, in order to be distilled, the state $\left|\gamma_{n}, l\right\rangle\left|\phi_{0}\right\rangle$ should not undergo (or should undergo less than the other states) transitions to states with different master states $\left|\gamma_{m}, q\right\rangle\left|\phi_{j}\right\rangle, j \neq 0$. On the basis of this comment, each state $\left|\gamma_{n}, l\right\rangle$ may be thought of as a "channel" of probability loss, which may be opened or closed depend- 
ing on the specific features of $\hat{U}(\tau)$. If the channel is closed the relevant state is preserved, otherwise it is going to be lost.

\section{B. Quantum Zeno dynamics to control unitary survival probability: A toy model}

The survival probability of a physical system undergoing a unitary time evolution may be changed using control mechanisms substantially relying on the dynamical quantum Zeno effect, i.e., quantum Zeno dynamics [9-11]. To better understand this fact, let us temporarily leave our original physical problem and consider a three-level system, assuming that its dynamics is governed by the tridiagonal Hamiltonian

$$
\Xi_{3}=\left(\begin{array}{ccc}
0 & \Omega & 0 \\
\Omega^{*} & 0 & \Lambda \\
0 & \Lambda^{*} & 0
\end{array}\right) \text {, }
$$

$\Omega$ being the coupling constant between the ground state and the first excited state $\left(|g\rangle\right.$ and $\left|e_{1}\right\rangle$, respectively), and $\Lambda$ being the coupling constant between the first and the second excited levels $\left(\left|e_{1}\right\rangle\right.$ and $\left\langle e_{2}\right\rangle$, respectively). We shall consider the dynamics in the regime $\Lambda=0$ as the unperturbed one, and it is straightforward to prove that it corresponds to Rabi oscillations between $|g\rangle$ and $\left|e_{1}\right\rangle$. The second excited state $\left|e_{2}\right\rangle$ is stationary. The introduction of the second coupling $\Lambda \neq 0$ gives rise to a modified regime, known as Zeno dynamics, such that for $|\Lambda| \gg|\Omega|$ the dynamics of the ground state is hindered. More in detail, assuming that the system starts in the state $|g\rangle$, the relevant survival probability is given by

$$
\wp_{g}\left[\Xi_{3}, \tau\right] \equiv\left|\left\langle g\left|e^{-i \Xi_{3} \tau}\right| g\right\rangle\right|^{2}=\left[\frac{|\Lambda|^{2}+|\Omega|^{2} \cos (\omega \tau)}{|\Lambda|^{2}+|\Omega|^{2}}\right]^{2}
$$

with $\omega=\sqrt{|\Omega|^{2}+|\Lambda|^{2}}$.

Looking at this formula it is easy to see that in the regime $|\Lambda| \gg|\Omega|$, the probability approaches unity, $\wp_{g} \approx 1$, meaning that the unperturbed dynamics is hindered by the action of the $\Lambda$ coupling which may be interpreted as a measurement coupling. Indeed the transitions from $\left|e_{1}\right\rangle$ to $\left|e_{2}\right\rangle$ produce in some sense a way to measure the population of level $\left|e_{1}\right\rangle$ [10-12], putting this behavior in the framework of quantum Zeno effect. Apart from philosophical and semantical discussions, the effect of the second (strong) coupling is to hinder the dynamics due to the first coupling.

The scheme may be generalized, assuming, for example, that a third excited level $\left|e_{3}\right\rangle$ coupled with the second exists, as expressed by the Hamiltonian

$$
\Xi_{4}(\Omega, \Lambda, \Gamma)=\left(\begin{array}{cccc}
0 & \Omega & 0 & 0 \\
\Omega^{*} & 0 & \Lambda & 0 \\
0 & \Lambda^{*} & 0 & \Gamma \\
0 & 0 & \Gamma^{*} & 0
\end{array}\right) .
$$

It has been shown that, in the regime $|\Gamma| \gg|\Lambda|$, the hindering effect due to the $\Lambda$ coupling is canceled $[10,18]$. In fact, we have

$$
\wp_{g}\left[\Xi_{4}, \tau\right] \equiv\left|\left\langle g\left|e^{-i \Xi_{4} \tau}\right| g\right\rangle\right|^{2} \approx \cos ^{2}(|\Omega| \tau),
$$

the unperturbed dynamics being restored.

Let us summarize this phenomenology. The four-level system under scrutiny performs Rabi oscillations between the two lowest states (those coupled by $\Omega$ ) when $\Lambda=\Gamma=0$. Such a dynamical regime corresponds to the unperturbed time evolution. As $\Lambda$ is made nonvanishing, the dynamics becomes more and more complicated until the condition $|\Lambda| \gg|\Omega|$ is reached. In such a situation, the dynamics of the lowest level is frozen, and this is just the continuous measurement quantum Zeno effect already recalled. Once $\Gamma$ is also adjusted as a nonvanishing coupling constant, an unexpected phenomenon does happen: as $|\Gamma|$ grows up, the effect due to the strong coupling $\Lambda$ between the second and the third levels becomes relatively weaker and weaker, up to the point, identified by the condition $|\Gamma| \gg|\Lambda|$, wherein the original Rabi oscillations are completely restored. In agreement with Refs. $[10,18]$, one can interpret $\Lambda$ and $\Gamma$ couplings as "continuous" measurements so that it is possible to give the following metaphorical statement: "a watched pot never boils" $(|\Lambda| \gg \Omega, \Gamma=0)$ but "a watched cook can freely watch a boiling pot" $(|\Lambda| \gg|\Omega|,|\Gamma| \gg|\Lambda|)[10]$.

In passing, we mention the fact that the hierarchical chain of interactions in principle may be extended (with further rings), maintaining the same substantial features: the last ring of the chain is able to destroy the effects of the previous one depending on how the relevant coupling strengths compare to each other. For instance we could add a fifth level and a fourth coupling involving the fourth and fifth levels. In this way, we obtain a $5 \times 5$ tridiagonal matrix. In concomitance with an increase of the fourth coupling strength, the hindering of inhibition given by the third coupling $(\Gamma)$ is hindered.

\section{Low-, high-, and bandpass bosonic filters}

Let us now come back to our original framework and consider the case when the master system is a three-level system and the slave is a harmonic oscillator. Assume that the unitary $M-S$ evolution is induced by the Hamiltonian

$$
\begin{aligned}
\hat{H}_{\Lambda}= & \Omega\left[f_{p}^{g 1}\left(\hat{a}^{\dagger} \hat{a}\right) \hat{a}^{p}\left|e_{1}\right\rangle\langle g|+\text { H.c. }\right]+\Lambda\left[f_{q}^{12}\left(\hat{a}^{\dagger} \hat{a}\right) \hat{a}^{q}\left|e_{2}\right\rangle\left\langle e_{1}\right|\right. \\
& + \text { H.c. }],
\end{aligned}
$$

where $p$ and $q$ are integer numbers related to the specific choice of the laser frequencies (sidebands), $\Omega$ and $\Lambda$ the (real) coupling constants, $\hat{a}$ the harmonic-oscillator annihilation operator (here the notation is such that $\hat{a}^{-s} \equiv \hat{a}^{\dagger}$ for positive $s$ ), while $|g\rangle,\left|e_{1}\right\rangle$, and $\left|e_{2}\right\rangle$ are the three atomic states effectively involved in the dynamics. Finally the functions (assumed to be real, for notational simplicity) $f_{p}^{g 1}$ and $f_{q}^{12}$ are introduced.

Consider the unitary evolution due to $\hat{H}_{\Lambda}$ in Eq. (18) in the special regime $\Lambda=0$ and $p=0$. Then the repeated detections of the atomic state $|g\rangle$ lead to the standard quantum nondemolition measurements, by which it is possible to extract, i.e., distill, a number state for instance in the context of trapped ions and cavity QED [6,7]. More in detail, denoting by $|n\rangle$ the harmonic-oscillator Fock states, the effective non- 
unitary evolution operator acting upon the vibrational state is given by

$$
\begin{aligned}
\hat{V}_{\Lambda=0}(\tau) & \equiv\left\langle g\left|e^{-i \hat{H}_{\Lambda=0} \tau}\right| g\right\rangle=\cos \left[\Omega f_{0}^{g 1}\left(\hat{a}^{\dagger} \hat{a}\right) \tau\right] \\
& =\sum_{n=0}^{\infty} \cos \left[\Omega f_{0}^{g 1}(n) \tau\right]|n\rangle\langle n| .
\end{aligned}
$$

Since in this case the Fock states are eigenstates of $\hat{V}(\tau)$, we have $\left|\gamma_{n}, l\right\rangle:=|n\rangle$. It is possible to choose $\tau=\tau_{\bar{n}}$ such that $\Omega f_{0}^{g 1}(\bar{n}) \tau_{\bar{n}}=\pi$. Assuming that for $n \neq \bar{n}, f_{0}^{g 1}(n)$ and $f_{0}^{g 1}(\bar{n})$ are incommensurable, the eigenvector $|\bar{n}\rangle \otimes|g\rangle$, and it only is preserved by the distillation procedure $\left(\left|\cos \left[\Omega f_{0}^{g 1}(\bar{n}) \tau_{\bar{n}}\right]\right|=1\right)$, while all the others are partially destroyed at each masterstate detection $\left(\left|\cos \left[\Omega f_{p}^{g 1}(n) \tau_{\bar{n}}\right]\right|<1\right.$ for $\left.\left.n \neq \bar{n}\right)\right)$. In the context of trapped ions a similar approach is used to realize quantum nondemolition measurements $[6,7]$.

In the case of positive $p(p>0)$, it is possible to realize what is called a low-pass bosonic filter. In fact, one easily obtains

$$
\hat{V}_{\Lambda=0}(\tau)=\sum_{n=0}^{p-1}|n\rangle\left\langle n\left|+\sum_{n=p}^{\infty} \cos \left[\Omega_{n} \tau\right]\right| n\right\rangle\langle n|,
$$

with $\Omega_{n} \equiv \Omega f_{p}^{g 1}(n-p) \sqrt{n ! /(n-p) !}$. Unless $\Omega_{n} \tau=l \pi$ for any $n$ and integer $l$, one realizes the projector $\sum_{n=0}^{p-1}|n\rangle\langle n|$ that filters those bosonic states characterized by excitation numbers lower than $p$. In the context of trapped ions, a strategy based on a coupling of the form in Eq. (18) with $p=1$ is used to realize the so-called resolved sideband cooling $[6,14]$.

Consider now the effect of the freezing agent, i.e., assume that $\Lambda \neq 0$. In this case the total Hamiltonian $\hat{H}_{\Lambda}$ is substantially characterized by the three-dimensional invariant subspaces $\left\{|n\rangle \otimes|g\rangle,|n-p\rangle \otimes\left|e_{1}\right\rangle,|n-p-q\rangle \otimes\left|e_{2}\right\rangle\right\}$, wherein the operator may be represented as a $3 \times 3$ block of the form

$$
\Xi_{n}=\left(\begin{array}{ccc}
0 & \Omega_{n} & 0 \\
\Omega_{n}^{*} & 0 & \Lambda_{n} \\
0 & \Lambda_{n}^{*} & 0
\end{array}\right),
$$

with $\Omega_{n}=\Omega f_{p}^{g 1}(n-p) \sqrt{n ! /(n-p) !}$ as before and $\Lambda_{n}=\Lambda f_{q}^{12}(n$ $-p-q) \sqrt{(n-p) ! /(n-p-q) !}$. Of course, depending on $p$ and $q$, which are assumed to be non-negative here, there could exist also invariant doublets and singlets. For instance, in correspondence to $n-p<0$ there are singlets, while if $n-p$ $\geqslant 0$ and $n-p-q<0$ there are doublets.

It is straightforward to evaluate the nonunitary operator $\hat{V}_{\Lambda} \equiv\left\langle g\left|e^{-i \hat{H}_{\Lambda}}\right| g\right\rangle$. In particular, in the case $p=0$ (previously analyzed in the absence of a freezing agent) and positive $q$, we obtain

$$
\begin{aligned}
\hat{V}_{\Lambda}(\tau)= & \sum_{n=0}^{q-1} \cos \left[\Omega f_{0}^{g 1}(n) \tau\right]|n\rangle\langle n| \\
& +\sum_{n=q}^{\infty} \frac{\left|\Lambda_{n}\right|^{2}+\left|\Omega_{n}\right|^{2} \cos \left(\omega_{n} \tau\right)}{\left|\Lambda_{n}\right|^{2}+\left|\Omega_{n}\right|^{2}}|n\rangle\langle n|
\end{aligned}
$$

with $\omega_{n}=\sqrt{\left|\Lambda_{n}\right|^{2}+\left|\Omega_{n}\right|^{2}}$. The operator is diagonal in the Fock basis.

In the dynamical regime characterized by $\Lambda \gg \Omega, p=0$, if we assume that $f_{q}^{12}(n-q) \neq 0$ for all Fock states and that the functions $f_{0}^{g}, f_{q}^{12}$ are of the same order, an inequality $\left|\Lambda_{n}\right|$ $\gg\left|\Omega_{n}\right|$ holds $\forall n$. If the measurement interval $\tau$ is not fine tuned, i.e., $\tau \neq \tau_{0}^{(k)}, \tau_{1}^{(k)}, \ldots, \tau_{q-1}^{(k)}, \forall$ integer $k$, where $\tau_{j}^{(k)}$ satisfies $\Omega f_{0}^{g 1}(j) \tau_{j}^{(k)}=k \pi$, all Fock states with an excitation number less than $q$ are eliminated in the course of repeated measurements, hence realizing the projector $\hat{1}-\sum_{n=0}^{q-1}|n\rangle\langle n|$, that is, a high-pass filter:

$$
\left[\hat{V}_{\Lambda}(\tau)\right]^{N} \rightarrow \hat{1}-\sum_{n=0}^{q-1}|n\rangle\langle n| .
$$

We mention that in principle also a bandpass filter is realizable by considering a four-level system as the master. In fact, the Hamiltonian

$$
\begin{aligned}
\hat{H}_{\Lambda, \Gamma}= & \Omega\left[f_{p}^{g 1}\left(\hat{a}^{\dagger} \hat{a}\right) \hat{a}^{p}\left|e_{1}\right\rangle\langle g|+\text { H.c. }\right]+\Lambda\left[f_{q}^{12}\left(\hat{a}^{\dagger} \hat{a}\right) \hat{a}^{q}\left|e_{2}\right\rangle\left\langle e_{1}\right|\right. \\
& + \text { H.c. }]+\Gamma\left[f_{r}^{23}\left(\hat{a}^{\dagger} \hat{a}\right) \hat{a}^{r}\left|e_{3}\right\rangle\left\langle e_{2}\right|+\text { H.c. }\right],
\end{aligned}
$$

with $p=0$ and positive $q$ and $r$, yields, for $|\Gamma| \gg|\Lambda| \gg|\Omega|$, the nonunitary operator

$$
\begin{aligned}
\hat{V}_{\Lambda, \Gamma}(\tau) \approx & \sum_{n=0}^{q-1} \cos \left[\Omega f_{0}^{g 1}(n) \tau\right]|n\rangle\langle n| \\
& +\sum_{n=q}^{q+r-1} \frac{\left|\Lambda_{n}\right|^{2}+\left|\Omega_{n}\right|^{2} \cos \left(\omega_{n} \tau\right)}{\left|\Lambda_{n}\right|^{2}+\left|\Omega_{n}\right|^{2}}|n\rangle\langle n| \\
& +\sum_{n=q+r}^{\infty} \cos \left[\Omega f_{0}^{g 1}(n) \tau\right]|n\rangle\langle n| .
\end{aligned}
$$

The third sum in Eq. (25) involves just $\cos \left[\Omega f_{0}^{g 1}(n) \tau\right]$ because in the subspace generated by $\{|n\rangle, n=q+r, \ldots, \infty\}$ the unperturbed dynamics is restored due to the action of the third coupling $\Gamma_{n}\left(\left|\Gamma_{n}\right| \gg\left|\Lambda_{n}\right| \gg\left|\Omega_{n}\right|\right)$. Such a condition holds only in the subspaces wherein the third coupling is effective, that is, for $n \geqslant q+r$; indeed otherwise $\Gamma_{n}=0$.

Under the assumption

$$
\tau \neq \tau_{0}^{(k)}, \tau_{1}^{(k)}, \ldots, \tau_{q-1}^{(k)}, \tau_{q+r}^{(k)}, \ldots, \tau_{\infty}^{(k)},
$$

we obtain a bandpass filter

$$
\left[\hat{V}_{\Lambda, \Gamma}(\tau)\right]^{N} \stackrel{\text { large } N}{\rightarrow} \sum_{n=q}^{q+r-1}|n\rangle\langle n| .
$$

Although the condition in Eq. (26) may seem too strong, it actually is not. In fact, taking account of the fact that in practical situations, for instance in trapped ions and in cavity QED, one deals with bosonic states with a finite 
number of excitations, it would be sufficient to consider the condition up to a large but finite number $\bar{n}: \tau \neq \tau_{0}^{(k)}, \tau_{1}^{(k)}, \ldots, \tau_{q-1}^{(k)}, \tau_{q+r}^{(k)}, \ldots, \tau_{\bar{n}}^{(k)}$.

\section{APPLICATIONS IN TRAPPED IONS AND CONCLUDING REMARKS}

Some of the applications reported in the previous sections may be realized in the context of trapped ions. As is well known, a time-dependent quadrupolar electric field is able to confine a charged particle, providing an effective quadratic potential that induces a harmonic motion $[19,20]$. When the confined particle is an ion, the complete result is a compound system possessing both fermionic and bosonic degrees of freedom, the first ones describing the internal motion of the electrons with respect to the atomic nucleus, the second ones describing the ion center-of-mass motion. In most of the experiments, only a few atomic states are really involved in the dynamics and a single vibrational mode is considered. Following Ref. [21], it is possible to realize an experimental setup which involves, in the dynamics, only the following three atomic levels of ${ }^{9} \mathrm{Be}^{+}:|g\rangle:=\left|{ }^{2} S_{1 / 2}, F=1, m_{F}=1\right\rangle,\left|e_{1}\right\rangle$ $:=\left|{ }^{2} S_{1 / 2}, F=2, m_{F}=2\right\rangle, \quad\left|e_{2}\right\rangle:=\left|{ }^{2} S_{1 / 2}, F=2, m_{F}=1\right\rangle$, using a magnetic field of $1 \mathrm{mT}$ to obtain the useful level splittings $\left[\omega\left(e_{2}\right)-\omega\left(e_{1}\right) \approx 100 \mathrm{MHz}, \omega\left(e_{1}\right)-\omega(g) \approx 1 \mathrm{GHz}\right]$, and exploiting the auxiliary level $\left|{ }^{2} P_{1 / 2}, F=2, m_{F}=2\right\rangle$ to realize Raman coupling schemes.

Consider now the action of two effective (i.e., implemented via Raman schemes) lasers, one tuned to the $p$ th red sideband of the atomic transition $|g\rangle \rightarrow\left|e_{1}\right\rangle$, and the other to the $q$ th red sideband of the atomic transition $\left|e_{1}\right\rangle \rightarrow\left|e_{2}\right\rangle$. The relevant interaction-picture Hamiltonian in the rotating wave approximation is given by $[15,21]$

$$
\begin{aligned}
\hat{H}_{\Lambda}= & \Omega\left[f_{p}\left(\hat{a}^{\dagger} \hat{a}, \eta_{1}\right) \hat{a}^{p}\left|e_{1}\right\rangle\langle g|+\text { H.c. }\right]+\Lambda\left[f_{q}\left(\hat{a}^{\dagger} \hat{a}, \eta_{2}\right) \hat{a}^{q}\left|e_{2}\right\rangle\left\langle e_{1}\right|\right. \\
& + \text { H.c. }],
\end{aligned}
$$

where integers $p$ and $q$ are related to the specific choice of the laser frequencies (sidebands), the coupling constants $\Omega$ and $\Lambda$ are proportional to the laser intensities, the relevant Lamb-Dicke parameters $\eta_{j}(j=1,2)$ express the ratio between the vibrational ground state oscillation amplitudes and the laser wavelengths, and $\hat{a}$ is the harmonic-oscillator annihilation operator (here the notation is the same as before). The functions $f_{p}$ and $f_{q}$ express nonlinear vibrational energy dependence of vibronic couplings [15],

$$
\begin{aligned}
f_{s}\left(\hat{a}^{\dagger} \hat{a}, \eta\right) & \equiv e^{-\eta^{2} / 2} \sum_{l=0}^{\infty} \frac{(i \eta)^{2 l}}{l !(l+s) !} \hat{a}^{\dagger l} \hat{a}^{l} \\
& =e^{-\eta^{2} / 2} \sum_{n=0}^{\infty} \frac{n !}{(n+s) !} L_{n}^{(s)}\left(\eta^{2}\right)|n\rangle\langle n|,
\end{aligned}
$$

where $L_{n}^{(s)}\left(\eta^{2}\right)$ are Laguerre polynomials and are such that for very small Lamb-Dicke parameters [the so-called LambDicke limit (LDL)], they almost approach unity, while for larger values of $\eta$ they exhibit a strong nonlinearity in the variable $\hat{a}^{\dagger} \hat{a}$, and possess some zeros. In the LDL, the con- dition $|\Lambda| \gg|\Omega|$ yields the inequality $\left|\Lambda_{n}\right| \gg\left|\Omega_{n}\right|$, making possible the realization of the high-pass filter.

In conclusion, in this paper we have addressed the general problem of how to project a quantum system in a prefixed subspace, or, in other words, we have described a way to realize the action of a projection operator. We have utilized the distillation approach based upon repeated measurements on a part of a bipartite system [3]. Moreover we have taken account of the possibility of exploiting the generalized quantum Zeno effect, or quantum Zeno dynamics, as a control mechanism of the two-subsystem-interaction determining the target subspace. Our main result is therefore that the presence of such a control mechanism transforms the original distillation process into a driven distillation. This allows the realization of what we have called high-, low-, and bandpass bosonic filters, that is, the realization of projection operators on subspaces whose states possess excitation numbers higher or lower than a quantity, or in a range, respectively.

Concerning the efficiency of the process, we stress the fact that the distillation here reported through all the applications considered (satisfying the condition $\gamma=1$ ) practically realizes (simulates) a measurement on the slave projecting the slave system onto a subspace with a probability, given by Eq. (7), which is just the probability of finding the target slave subspace in the initial slave state. Therefore, the efficiency of the procedure is not improved since it is intrinsically related to the initial condition of the system. Our analysis has indeed the merit of providing a more transparent understanding of the process, clarifying the relation with the survival probabilities, and hence the possibility of governing it.

We conclude by emphasizing that the method and the idea presented in this paper are general and exploitable in different physical contexts for both fundamental and technological research.

\section{ACKNOWLEDGMENTS}

This work is partly supported by the bilateral ItalianJapanese Project No. $15 \mathrm{C} 1$ on "Quantum Information and Computation" of the Italian Ministry for Foreign Affairs, by a Grant for the 21st Century COE Program (Physics of SelfOrganization Systems) at Waseda University and a Grant-inAid for Priority Areas Research (B) (No. 13135221), both from the Ministry of Education, Culture, Sports, Science and Technology, Japan, and by a Grant-in-Aid for Scientific Research (C) (No. 14540280) from the Japan Society for the Promotion of Science. Moreover, the authors acknowledge partial support from Palermo University in the context of the bilateral agreement between Palermo University and Waseda University.

\section{APPENDIX: EFFICIENCY}

In this appendix we prove the limit expressed by Eq. (7). Assume that the system is prepared, for simplicity, in the pure state $\left|\psi_{0}\right\rangle=|\varphi\rangle\left|\phi_{0}\right\rangle,|\varphi\rangle$ being the initial slave state. The probability of finding the master system in $\left|\phi_{0}\right\rangle$ after the unitary $M-S$ interaction is $\wp_{1}^{(M)}=\left\|\left\langle\phi_{0}\left|e^{-i H \tau}\right| \psi_{0}\right\rangle\right\|^{2}$ 
$=\| \hat{V}(\tau)|\varphi\rangle \|^{2}$, where $\|\cdot\|^{2} \equiv|\langle\cdot \mid \cdot\rangle|^{2}$ denotes the relevant Hilbert space norm. The collapsed state is $\left|\psi_{1}\right\rangle=\left(1 / \sqrt{\wp_{1}}\right)$ $\times[\hat{V}(\tau)|\varphi\rangle]\left|\phi_{0}\right\rangle$. After the next unitary interaction and measurement one obtains $\wp_{2}^{(M)}=\left\|\left\langle\phi_{0}\left|e^{-i \hat{H} \tau}\right| \psi_{1}\right\rangle\right\|^{2}$ $=\left(1 / \wp_{1}^{(M)}\right) \|\left[\hat{V}^{2}(\tau)|\phi\rangle\right] \|^{2}, \quad$ and $\quad\left|\psi_{2}\right\rangle=\left(1 \sqrt{\wp_{1}^{(M)} \wp_{2}^{(M)}}\right)$ $\times\left[\hat{V}^{2}(\tau)|\varphi\rangle\right]\left|\phi_{0}\right\rangle$.

In general the probability $\wp_{n}^{(M)}$ is given by

$$
\wp_{n}^{(M)}=\frac{1}{\wp_{0}^{(M)} \wp_{1}^{(M)} \cdots \wp_{n-1}^{(M)}} \| \hat{V}^{n}(\tau)|\varphi\rangle \|^{2}
$$

and the state $\left|\psi_{n}\right\rangle$ reads as

$$
\left|\psi_{n}\right\rangle=\frac{1}{\sqrt{\wp_{0}^{(M)} \wp_{1}^{(M)} \cdots \wp_{n}^{(M)}}} \hat{V}^{n}|\varphi\rangle\left|\phi_{0}\right\rangle .
$$

From Eq. (A1) it follows that

$$
\prod_{k=1}^{N} \wp_{k}^{(M)}=\wp_{N}^{(M)} \prod_{k=1}^{N-1} \wp_{k}^{(M)}=\| \hat{V}^{N}(\tau)|\varphi\rangle \|^{2} .
$$

Considering large enough $N$, corresponding to which $\hat{V}^{N}(\tau)$ $\rightarrow \gamma^{N} \hat{R}^{N} \hat{P}_{\text {dist }}$ [compare with Eq. (5)], we easily obtain

$$
\prod_{k=1}^{N} \wp_{k}^{(M)} \approx \gamma^{N} \| \hat{P}_{\mathrm{dist}}|\varphi\rangle \|^{2}
$$

which in the case $\gamma=1$ expresses the same content as that of Eq. (7).

The result is of course the same as Eq. (7) even if we start with a nonpure state.
[1] C. H. Bennett et al., Phys. Rev. Lett. 76, 722 (1996); 78, 2031(E) (1997); Phys. Rev. A 54, 3824 (1996); J. I. Cirac, A. K. Ekert, and C. Macchiavello, Phys. Rev. Lett. 82, 4344 (1999). See also Refs. [2] and references therein.

[2] For reviews, see A. Galindo and M. A. Martin-Delgado, Rev. Mod. Phys. 74, 347 (2002); The Physics of Quantum Information, edited by D. Bouwmeester, A. Ekert, and A. Zeilinger, (Springer, Berlin, 2000); M. A. Nielsen and I. L. Chuang, Quantum Computation and Quantum Information (Cambridge University Press, Cambridge, U.K., 2000).

[3] H. Nakazato, T. Takazawa, and K. Yuasa, Phys. Rev. Lett. 90, 060401 (2003).

[4] V. B. Braginsky and F. Ya. Khalili, Quantum Measurement (Cambridge University Press, Cambridge, U. K., 1992).

[5] D. F. Walls and G. J. Milburn, Quantum Optics (SpringerVerlag, Berlin, 1994).

[6] R. L. de Matos Filho and W. Vogel, Phys. Rev. Lett. 76, 4520 (1996).

[7] L. Davidovich et al., Phys. Rev. A 54, 5118 (1996).

[8] K. Wang et al., Phys. Rev. A 63, 043419 (2001).

[9] H. Nakazato, M. Namiki, and S. Pascazio, Int. J. Mod. Phys. B 10, 247 (1996); D. Home and M. A. B. Whitaker, Ann. Phys. (N.Y.) 258, 237 (1997).

[10] P. Facchi and S. Pascazio, Phys. Rev. Lett. 89, 080401 (2002).
[11] P. Facchi and S. Pascazio, Quantum Zeno and Inverse Quantum Zeno Effects, Progress in Optics vol. 42, edited by E. Wolf (Elsevier, Amsterdam, 2001), p. 147.

[12] B. Militello, A. Messina, and A. Napoli, Phys. Lett. A 286, 239 (2001).

[13] The most general case, in which the standard form (3) is no longer valid, owing to the presence of degenerate eigenvalues, is found in H. Nakazato, M. Unoki, and K. Yuasa, Phys. Rev. A 70, 012303 (2004).

[14] D. J. Wineland et al., J. Res. Natl. Inst. Stand. Technol. 103, 259 (1998).

[15] W. Vogel and S. Wallentowitz, in Coherence and Statistics of Photons and Atoms, edited by Jan Perina (Wiley, New York, 2001).

[16] D. Leibfried et al., Rev. Mod. Phys. 75, 281 (2003).

[17] B. Militello and A. Messina, Phys. Rev. A 70, 033408 (2004).

[18] B. Militello, A. Messina, and A. Napoli, Fortschr. Phys. 49, 1041 (2001).

[19] P. K. Ghosh, Ion Traps (Clarendon Press, Oxford 1995).

[20] P. E. Toschek, in New Trends in Atomic Physics, edited by G. Grynberg and R. Stora (Elsevier, Amsterdam, 1984), p. 282.

[21] S. Maniscalco and A. Messina, Fortschr. Phys. 49, 1027 (2001). 